\title{
INFRARED OBSERVATIONS OF GALACTIC PLANETARY NEBULAE
}

\author{
A. Preite-Martinez \\ Istituto di Astrofisica Spaziale \\ C.P. 67 \\ 00044 Frascati, Italy
}

\section{INTRODUCTION}

Five months after the 1982 Symposium in London the InfraRed Astronomical Satellite (IRAS) was launched. Undoubtedly this has been the major event of the last five years in the infrared world, with a great impact in the field of Planetary Nebulae (PN) research $\left({ }^{\wedge}\right)$.

In the following, IRAS observations of PN will be presented and discussed, as well as ground based observations. The presentation and discussion of the data will highligt the general properties of the infrared emission of PN, with particular emphasis on the spatial distribution of the IR emission, on the energy distribution over the range 1 to 100 micron, and on some of the relevant properties of dust and ionized gas emission features.

The frequency range covered by IR observations is rather broad, and so is the number of questions we can address ourselves and try to answer with the help of IR data. A comprehensive list of all possible problems is out of the scope of this review, and is covered also by other reviews in this volume. Nonetheless it is worth stressing two points connected mainly with the evolutionary scenario : with infrared observations we can shed light on the link between asymptotic giant branch stars and nuclei of planetary nebulae, and whether dust properties are related to basic parameters of the progenitor star.

IRAS photometric and spectral data, and ground-based photometric observations of various kind will be presented and discussed in sections 2 and 3, respectively.

\section{IRAS OBSERVATIONS}

We know that planetary nebulae are strong sources of emission in the far infrared (FIR) since 20 years (Gillet et al. 1967), but until 1983 only some of the brightest PN were observed in the near IR and 10 micron windows, and very few in the FIR. With IRAS we had the first survey in the FIR covering 98\% of the sky, but missing one of the most interesting and brightest objects: NGC 7027.

The three onboard instruments were: (i) a Survey Array operating in four bands centered at 12, 25, 60 , and $100 \mu \mathrm{m}$ with a rectangular field of view (FOV) ranging from $0.75 \times 4.5$ to $3 \times 5$ arcmin; (ii) a Chopped Photometric Channel operating at 50 and $100 \mu \mathrm{m}$ with circular FOV of 1.2 arcmin; and (iii) a Low Resolution Spectrometer operating between 7.5 and $23 \mu \mathrm{m}$ with FOV in the range 5 to 7.5 arcmin and resolution between 14 and 35 .

$(\wedge)$ The Infrared Astronomical Satellite (IRAS) was developed and was operated by NIRV, NASA, and SERC. 
Three main data products are now available: (i) the Survey data on point sources, collected in the Point Source Catalogue (PSC), (ii) an Atlas of low resolution spectra (Olnon and Raimond, 1986), and (iii) Skyflux maps. Other products, as the Small Scale Structure Catalogue (SSSC), are also available. Further details can be found in the IRAS Explanatory Supplement (Beichman et al.,1985).

IRAS observations of planetary nebulae are also discussed in Pottasch et al.(1984a), Pottasch (1986, 1987), Kwok et al.(1986), and Iyengar (1986).

\subsection{Maps}

One of the point to bear in mind is that flux densities for objects larger than about $2 \times 4$ arcmin are severely underestimated (if present at all) in the PSC. Leene and Pottasch (1987a) derived maps of NGC 7293, the Helix nebula, in order to estimate the total energy emitted by the nebula and give an indication of the origin of the total emission. The four panels of Figure 1 show the infrared data of NGC 7293 for $12,25,60$, and $100 \mu \mathrm{m}$. The maps at different wavelengths show a totally different structure. It is clear that emission from dust is unable to explain the different morphologies observed. They show that ion line emission plays an important role at 12 and $25 \mu \mathrm{m}$. Indeed, the $12 \mu \mathrm{m}$ emission can be entirely attributed to the S IV $10.52 \mu \mathrm{m}$ and possibly to Ne II $12.81 \mu \mathrm{m}$, while the centrally peaked $25 \mu \mathrm{m}$ emission is attributed to the O IV $25.87 \mu \mathrm{m}$ line. Only the 60 and $100 \mu \mathrm{m}$ bands are dominated by dust emission.

The effect of line emission on the IRAS data of planetaries has been also pointed out by PreiteMartinez and Pottasch (1987) and Leene and Pottasch (1987b).

Another important result is the determination of integrated flux densities. In order to show how standard IRAS products can underestimate fluxes for large objects, in Table 1 we compare the integrated flux densities for NGC 7293 as derived by Leene and Pottasch (1987a) with flux densities listed in IRAS catalogues (PSC and SSSC).

Table 1.

Flux densities of NGC 7293 (Jy).

Band from map PSC SSSC

$\begin{array}{llcr}12 & 11.3 & - & - \\ 25 & 18.5 & - & 6 \\ 60 & 179 . & 1.6 & 56 \\ 100 & 406 . & 12 . & -\end{array}$

Figure 1. IR maps of NGC 7293 from Leene and Pottasch (1987a).

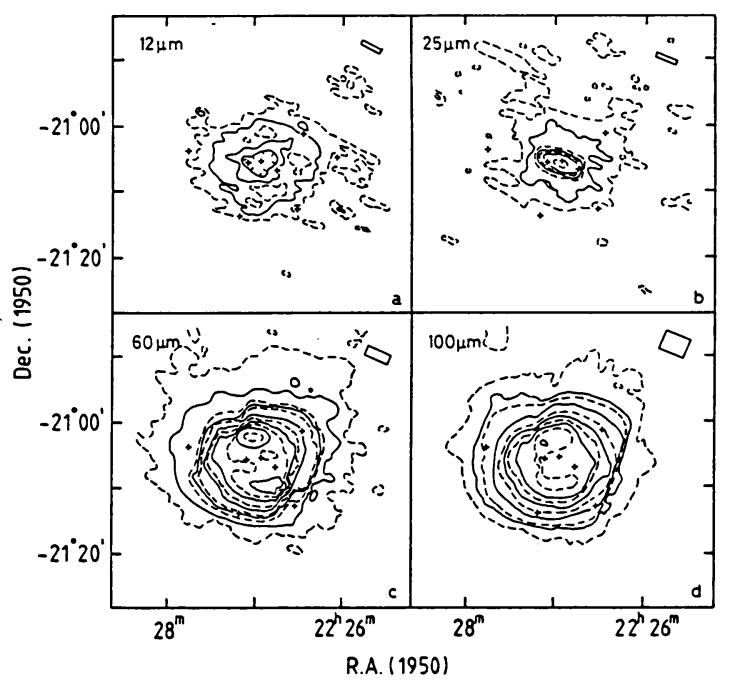

A similar analysis has been done by Zhang et al.(1987) for NGC 6853 and it is in progress on other extended nebulae, as A 21. 


\subsection{Search for IR halos}

During the IRAS mission a sample of $67 \mathrm{PN}$ were observed in the pointing mode by Leene et al. (1987). The nebulae were selected for their large optical diameter and/or the presence of multiple rings and halos. Also objects smaller than the beam size were selected to search for the presence of weak FIR halos. It is important to know if a source is extended or has some kind of halo showing up in the IR, because the spatial distribution of IR emission can shed light on the origin and evolution of the dust shell, as discussed by Kwok (1980), Natta and Panagia (1981), and Lenzuni et al.(1987).

From the analysis of their sample of PN Leene et al. (1987) find that FIR emission usually originates in the ionized region. This is particularly true for old, optically thin nebulae. On the contrary, for BD+303639 and NGC 6543, two relatively young nebulae, there is clear evidence for dust emission well outside the ionized region. It should be mentioned though that far-IR slit scans of NGC 7027 at 50 and 100 microns taken by Lester et al. (1986) from the Kuiper Airborne Observatory show that the spatial distribution of the far-IR emission corresponds very closely with the distribution of ionized gas.

\subsection{IRAS spectra}

There are several advantages over the optical and UV lines in using infrared lines: (i) some of the ions are only observed in the IR; therefore they are very useful in determining "total" abundances; (ii) intensities are less sensitive to electron temperature because of the small excitation energy involved, and they are also less influenced by extinction; (iii) we can derive total line intensities for PN with sizes up to about 40", while smaller diaphragms are used from the ground in order to minimize sky background.

On the other hand we should bear in mind that the reliability of IRAS spectral data is rather uneven, both for line intensities and continuum levels. Line fluxes and continuum intensities can vary up to a factor of 2 from one spectrum to another. Sometimes features present in the averaged spectrum appear in only one of the individual spectra. Moreover, as the sources become more extended than 40", the resolution of the spectrometer is degraded: this is why some bright, but large, nebulae have not been measured.

Recently Pottasch et al.(1984b, 1985, 1986) presented and discussed more than 60 LRS spectra of PN. They found that the relative importance of ionic lines and continuum emission is strongly variable from object to object, and that many lines of $\mathrm{Ne}, \mathrm{S}$, and $\mathrm{Ar}$ are strong enough to be detected. Seven lines were measured, Ne III 15.5 and Ne VI $7.65 \mu \mathrm{m}$ for the first time. The ratio of IR to optical lines of NeIII, $\mathrm{NeV}$ and S III were used to derive electron temperatures and densities. The determination of Te and ne from the Ne III and S III ratios agrees very well with values found from optical line ratios. On the other hand the values of the density found from the $\mathrm{Ne} \mathrm{V}$ ratio are an order of magnitude higher, indicating a higher density in the higly ionized regions near the centre of the nebula. More reliable total abundances of $\mathrm{Ne}$ and $\mathrm{S}$ were derived, in particular for Ne, where in many nebulae no correction for missing stages of ionization had to be made.

\subsection{The IRAS Point Source Catalogue}

More than 900 PSC entries are associated with objects listed in the P-K catalog of planetary nebulae (Perek and Kohoutek, 1976), but not all PN known today were listed in the P-K catalogue, and not all the sources in P-K are PN (see Acker et al. 1987). In Table 2 we list the number of PSC object associated with planetary nebulae, with additional information on the quality of the detection and galactic distribution. At point 4, "NoVar" and "NoConf" mean that the variability index is lower than $20 \%$, and that the confusion flag is set to 0 if flux quality is $>1$, respectively. The number of misclassified PN is also indicated ("noPN"). 
Table 2: PSC objects associated with $P-K$ sources

Sample

1) Associated

2) Detected

(Flux Quality $\geq 2,2,2,2$ )

3) Detected

(Flux Quality $\geq 1,2,2,1$ )

" " " F12/F25 < 1

4) Detected, NoVar, NoConf

" F12/F25 $<1$

" $|1| \leq 15^{\circ}$

" $\quad|1|>15^{\circ}$
Tot. $|\mathrm{b}| \leq 15^{\circ}$ noPN

$\begin{array}{lll}928 & 868 & 86 / 928\end{array}$

$168 \quad 148$

$\begin{array}{lll}685 & 642 \quad 39 / 685\end{array}$

577

$\begin{array}{lll}479 & 445 & 14 / 479\end{array}$

162

317
Figure 2. Colour-colour diagram for 685 planetaries with good IRAS detection (Flux Quality $\geq 1,2,2,1$ ).

Figure 3. Distribution of dust temperature for the same sample of 685 PN shown in Figure 2.
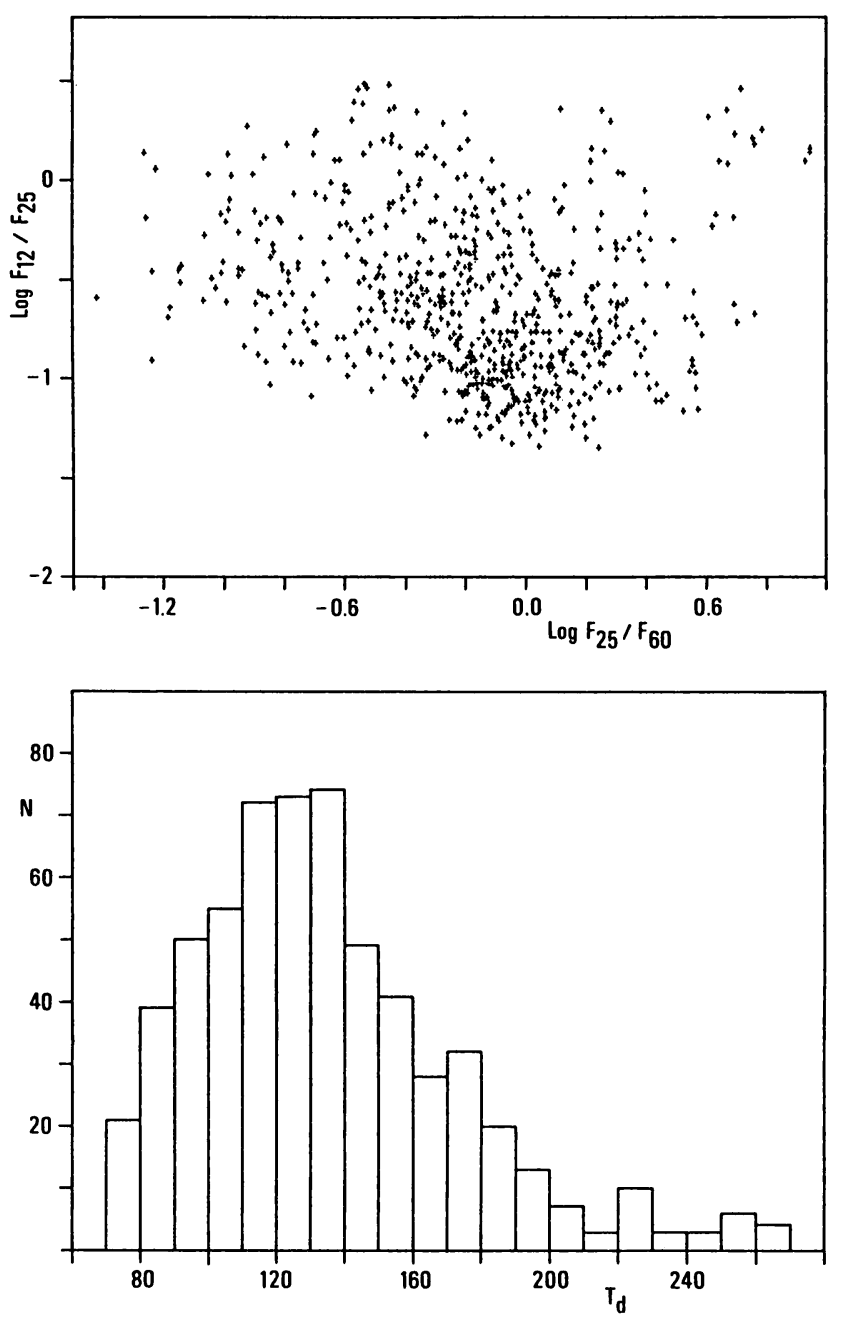
The 685 PK nebulae detected at least at 25 and 60 microns are shown in Figure 2 in the colourcolour plot involving the first three photometric IRAS bands. In Figure 3 the distribution of dust temperature for the same sample is also shown.

All nebulae show continuum emission in the far- $\mathbb{R}$, in excess of expected nebular continuum emission, due to dust heated to about $100-150 \mathrm{~K}$. The dust temperature $\mathrm{Td}$ is the parameter used to describe the distribution of emission. It is calculated between 25 and 60 microns, because the nebular flux usually peaks in this range.

Figure 4. Distribution of InfraRed Excess (IRE) for a sample of 159 PN selected from Pottasch et al. (1984a, 1987).

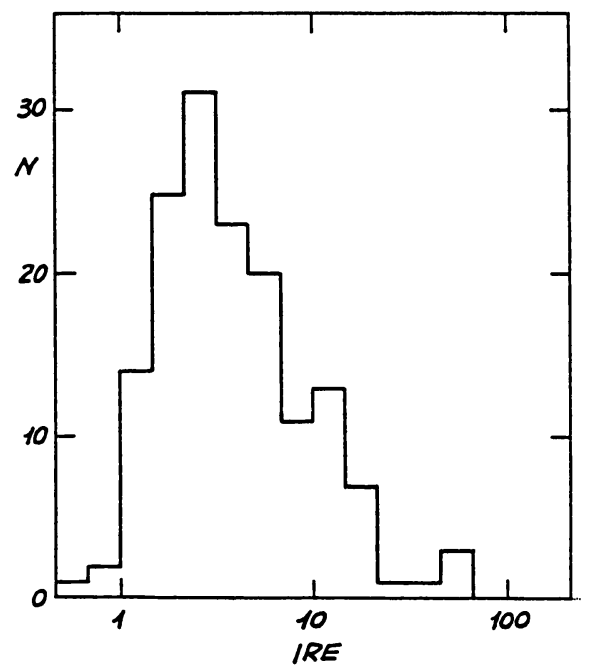

What is the origin of dust heating? As suggested by Krishna Swamy and O'Dell (1968) an important heating mechanism is scattering of Lyman- $\alpha$ photons generated in the ionized nebular gas, if the far-IR radiation comes from dust well mixed with the ionized gas. It is possible to calculate the IR flux expected if all Ly- $\alpha$ photons produced by recombinations in the nebula heat the dust, using the observed radio continuum emission to estimate the number of recombinations (Pottasch, 1984). We can then compare the expected IR flux F(IR) with the total observed IR flux and define as infrared excess (IRE) the ratio of observed total infrared emission to the energy available in Ly- $\alpha$ photons. In Figure 4 the distribution of the IRE is shown for a sample of 159 PN that includes galactic centre nebulae. Many authors (Pottasch, 1986, 1987; Pottasch et al. 1984a; Kwok et al. 1986; Iyengar 1986) have shown that the IRE is related to properties of the nebula: Ly- $\alpha$ heating is able to explain the amount of observed IR radiation, in particular in the case of large, old nebulae. Values of F(IR) slightly larger than expected could be explained by absorption of other strong nebular lines, as the CIV UV doublet. High values of the IRE $(>10)$ are found in compact, probably very young, nebulae. In these cases, heating by direct starlight is the most plausible cause of dust heating.

An interesting point is whether stellar radiation is absorbed directly by dust in the ionizing continuum, thus competing with the gas, or longward of the Lyman limit. Since large values of the IRE are usually found in nebulae excited by low temperature stars, probably the stellar radiation is absorbed by dust longward of the Lyman limit. This can be the case for ionization bounded nebulae surrounded by IR thermal emission due to heated dust. If ionizing photons are absorbed by dust, the determination of the stellar temperature can be affected, in particular when the Zanstra method is used: photons not absorbed by the gas will not be counted, thus the resulting temperature of the central star will be underestimated. Because of its different nature, the Energy-Balance method is much less affected. 


\section{GROUND-BASED OBSERVATIONS}

The success of the IRAS mission stimulated a reniewed interest in ground-based observations. A great number of planetary nebulae was observed in the near-, mid-IR from ground-based telescopes. In the following only photometric observations and spatial studies of selected nebulae will be discussed.

\subsection{Photometry}

More than 200 PN have been observed in the near-IR in recent years by Whitelock (1985), Kwok et al. (1986), Persi et al. (1987), and Preite-Martinez et al. (1987, private communication).

The importance of near-IR photometry is manyfold. First of all, it completes the information on the IR energy distribution. Secondly, it gives us a powerful tool for the classification of nebulae according to the principal source of emission. Furthermore, the presence of hot dust can be investigated.

Whitelock (1985) first pointed out that the near-IR two-colour diagram (J-H, H-K) can be used in order to classify PN according to their main source of emission. Three different sources can play a role. (i) Nebular emission : there can be a relevant contribution from thermal continuum due to $\mathrm{H}$ and $\mathrm{He}$ freefree and bound-free emission; two-photon emission can also contribute al low electron densities. Recombination lines of $\mathrm{H}$ (as $\mathrm{P} \beta, \mathrm{P} \gamma$, and $\mathrm{Br} \gamma$ ) and $\mathrm{He}$ (as $\mathrm{He} \mathrm{I} 1.083 \mu \mathrm{m}, 2.058 \mu \mathrm{m}$, and $\mathrm{He}$ II $1.162 \mu \mathrm{m}$ ) are expected to be strong features in this range. PN dominated by nebular (continuum and line) emission are classified as type N. (ii) Dust emission : cool dust, while dominating the emission in the far-IR, is not expected to contribute significantly in this range. Dust with temperatures $>800 \mathrm{~K}$ can be seen in the nearIR, if present. PN showing a substancial contribution of thermal emission by hot dust are classified as type D. (iii) Stellar continua : the Rayleigh-Jeans tail of the thermal emission from hot, bright central stars is sometimes seen, especially in the J band. A contribution from a cool companion of the central star can also show up in a few PN. In these cases nebulae are classified as type $S$.

The colours (J-H, H-K) of two large samples of PN are shown in the diagrams of Whitelock (1985, her Figure 1) and Persi et al. (1987, their Figure 1, here reproduced as Figure 5). It is clear from these diagrams that PN can be well separated and classified according to their near-IR colours. About half of

Figure 5. Near-IR two-colour diagram from Persi et al. (1987). Regions where different types of PN concentrate are indicated by the letters $N, D$, and $S$.

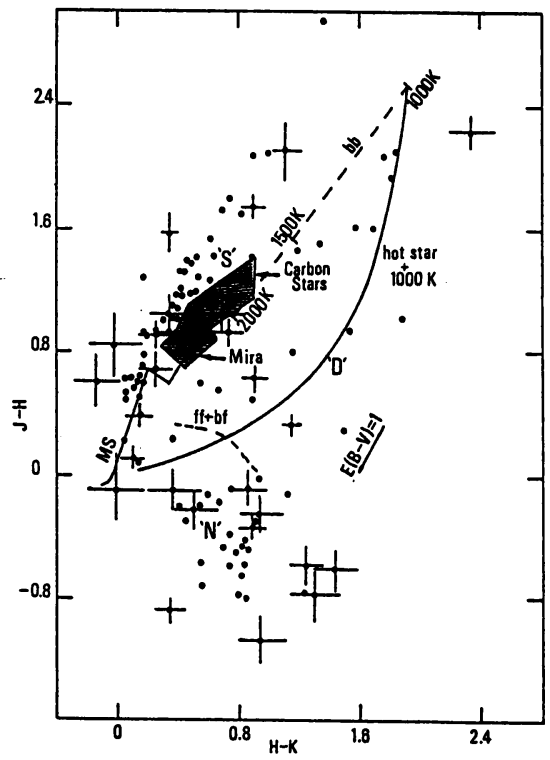


the nebulae of the total sample fall in a region called "nebular box" by Whitelock (1985). It can be roughly defined as the region of the two-colour diagram delimited by values of the observed $\mathrm{J}-\mathrm{H}<0.2$ and observed $\mathrm{H}-\mathrm{K}>0.3 \mathrm{mag}$.

Type D nebulae can be found above the nebular box and to the right of the cooler end of the main sequence. Type $S$ nebulae mostly fall in the region of Mira's and carbon stars. A significative fraction of the objects showing in Figure 5 the near-IR energy distribution of a cool main sequence star have been carefully reobserved. I have found that the majority of them were field stars and that the correct position for the objects is either in the $\mathrm{N}$ or $\mathrm{D}$ regions.

One of the most important point worth stressing about this near-IR classification scheme is that we can build up the two-colour diagram using observed magnitudes, regardless of extinction, with little loss in classification effectiveness. This is because the direction of the reddening correction is almost parallel to the black-body line and to the locus of $S$ type objects. In other words, it is impossible to transform a reddened $\mathrm{D}$ or $\mathrm{N}$ type object into a dereddened $\mathrm{S}$ type object, while dereddening $\mathrm{S}$ types only makes them hotter. Dereddened N sources do not change type, too. Such a feature of the (J-H, H-K) colours can also have practical applications in IR observing runs of planetaries.

If hot dust is present in the nebula, it is rather easy to recognize its signature in the two-colour diagram (H-K, K-L), although the $\mathrm{L}$ magnitude has been measured only for a sub-sample $(<30 \%)$ of nebulae. Objects of type S, N, and D are well separated also in this diagram (see Persi et al., 1987, their Figure 2). Sources of type D, as expected, and also many type N, show a distinct K-L excess with respect to stellar or nebular emission. It is not possible to ascertain whether the contribution to the $L$ band is due to thermal emission of hot dust or to emission features (or both) from photometric data alone. In order to give a more reliable indication on the nature of the emission processes involved, the spectrum and a thorough study of the IR energy distribution are required.

\subsection{Spatial studies}

Near-IR scanning (Phillips et al. 1984) and mapping techniques (Bentley et al. 1984; Lester and Dinerstein, 1984) were used in high angular resolution studies of a relatively small number $(\leq 20)$ of PN. Phillips et al. (1984) found in most cases that near-IR sizes were smaller than optical sizes. Only in NGC $6826,6891,7027$, and J900 dust and gas are probably well mixed. The most interesting result comes from IC 418: emission in band $\mathrm{H}$ originates from within the ionized zone, while $\mathrm{K}$ and $\mathrm{L}$ emissions extend outside the $\mathrm{H}$ II zone. From a detailed analysis of the spatial distribution of $\mathrm{K}$ and $\mathrm{L}$ emissions they inferred the presence of a circumnebular shell of dust with rather high temperatures and a strong temperature gradient. On the other hand Bentley et al. (1984) found that the observations of BD+303639 can be explained by the presence of two distinct species of dust, one of which is responsible for the emission features at 8.6 and $11.3 \mu \mathrm{m}$, possibly concentrated toward the edges of the nebula. Also in this case the observations are consistent with the dust being well mixed with the ionized gas.

Using a different but very promising technique, Arens et al. (1984) have performed observations of NGC 7027 with a two-dimensional charge-injection-device (CID) array operating at $10 \mu \mathrm{m}$. The $32 \times 32$ pixel CID array permits the mapping of extended sources at angular resolutions close to diffraction limits. The main result is that with a resolution $<2$ " radio maps and mid-IR maps are very similar, not only in their general appearance, but also in their details. This is the best evidence to date that the dust responsible for the emission in the $10 \mu \mathrm{m}$ window is coextensive and well mixed with the ionized gas.

Now that more sensitive and larger two-dimensional IR arrays are becoming available for groundbased as well as for the next generation of infrared satellites, the acquisition of high spatial and spectral images of PN will greatly help in the determination of the basic parameters of dust and gas IR emission, broadening our knowledge of the origin and properties of planetary nebulae. 


\section{References}

Acker.A., Chopinet,M., Pottasch,S.R., Stenholm,B.: 1987, Astron.Astrophys.Suppl.Ser. 71, 163

Arens,J.F., Lamb,G.M., Peck,M.C., Moseley,H., Hoffmann,W.F.,Tresch-Fienberg,R., Fazio,G.: 1984, Astrophys.J. 279, 685

Beichman,C.A., Neugebauer,G., Habing,H.J., Clegg,P.E., Chester,T.J.,eds. 1985, "Explanatory Supplement to the IRAS Catalogs and Atlases" (Washington:GPO)

Bentley,A.F., Hackwell,J.A., Grasdalen,G.L., Gehrz,R.D.: 1984, Astrophys.J. 278, 665

Gillet,F.C., Low,F.J., Stein,W.A.: 1967, Astrophys.J. Letters 149, L97

Iyengar,K.V.K.: 1986, Astron.Astrophys. 158, 89

Krishna Swamy,K.S., O'Dell,C.R.: 1968, Astrophys.J. Letters 151, L61

Kwok,S.: 1980, Astrophys.J. 236, 592

Kwok,S., Hrivnak,B.J., Milone,E.F.: 1986, Astrophys.J. 303, 451

Leene,A., Pottasch,S.R.: 1987a, Astron.Astrophys. 173, 145

Leene,A., Pottasch,S.R.: 1987b, "Planetary and Protoplanetary Nebulae: from IRAS to ISO", ed. A.Preite-Martinez (Reidel:Dordrecht),p.233

Leene,A., Zhang,C.Y., Pottasch,S.R.: 1987, Astron.Astrophys, in press

Lenzuni,P., Natta,A., Panagia,N.: 1987, "Planetary and Protoplanetary Nebulae: from IRAS to ISO", ed. A.Preite-Martinez (Reidel:Dordrecht), p.249

Lester,D.F., Dinerstein,H.L.: 1984, Astrophys.J. Letters 281, L67

Lester,D.F., Harvey,P.M., Joy,M.: 1986, Astrophys.J. 304, 623

Natta,A., Panagia,N.: 1981, Astrophys.J. 248, 189

Olnon,F.M., Raimond,E.: 1986, Astron.Astrophys.Suppl.Ser. 65, 607

Perek,L., Kohoutek,L.: 1967, "Catalogue of galactic planetary nebulae", Academ. Publish. House of the Czechoslovak Acad.of Sciences

Persi,P., Preite-Martinez,A., Ferrari-Toniolo,M., Spinoglio,L.: 1987, "Planetary and Protoplanetary Nebulae: from IRAS to ISO", ed. A.Preite-Martinez (Reidel: Dordrecht), p.221

Phillips,J.P., Sanchez Magro,C., Martinez Roger,C.: 1984, Astron.Astrophys. 133, 395

Pottasch,S.R.: 1984, "Planetary Nebulae", Reidel: Dordrecht

Pottasch,S.R.: 1986, "Light on dark matter", ed. F.P.Israel (Reidel:Dordrecht), p.131

Pottasch,S.R.: 1987, "Planetary and Protoplanetary Nebulae: from IRAS to ISO", ed. A.Preite-Martinez (Reidel:Dordrecht), p.1

Pottasch,S.R., Baud,B., Beintema,D.A., Emerson,J., Habing,H.J., Harris,S., Houck,J.R., Jenning, R.E., Marsden,P.: 1984a, Astron.Astrophys.138, 10

Pottasch,S.R., Beintema,D.A., Raimond,E., Baud,B., van Duinen,R., Habing,H.J., Houck,J.R., de Jong,T., Jenning,R.E., Olnon,F.M., Wesselius,P.R.: 1984b, Astrophys.J.Letters 278, L33

Pottasch,S.R., Preite-Martinez,A., Olnon,F.M., Raimond,E., Beintema,D.A., Habing,H.J.: 1985, Astron.Astrophys. 143, L11

Pottasch,S.R., Preite-Martinez,A., Olnon,F.M., Jing-Er,M., Kingma,S.: 1986, Astron.Astrophys. 161,363

Pottasch,S.R., Bignell,C., Olling,R., Zijlstra,A.A.: 1987, Astron.Astrophys. , in press

Preite-Martinez,A., Pottasch,S.R.: 1987, "Planetary and Protoplanetary Nebulae: from IRAS to ISO", ed. A.Preite-Martinez (Reidel:Dordrecht), p.197

Whitelock,P.A.: 1985, Mon. Not. Roy. Astron. Soc. 213, 59

Zhang,C.Y., Leene,A., Pottasch,S.R.: 1987, Astron.Astrophys. 178, 247 